\title{
Adaptação do índice de alimentação saudável ao guia alimentar da população brasileira'
}

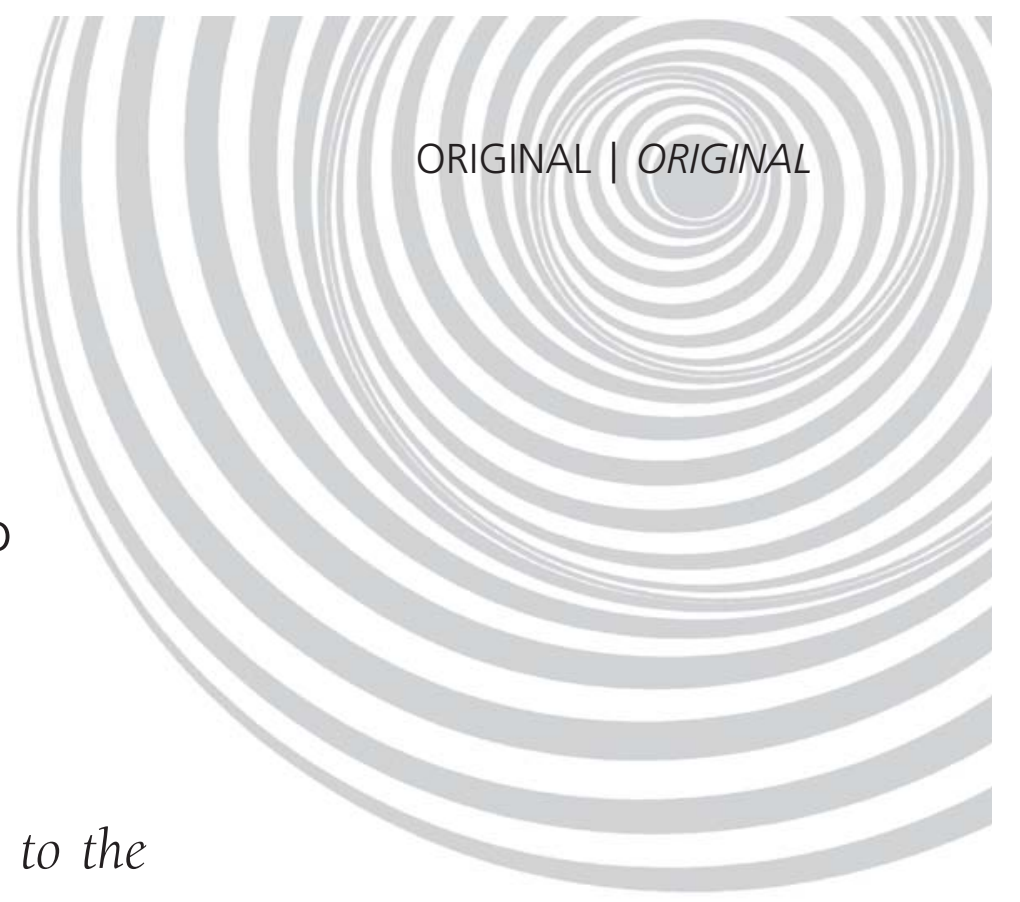

\section{Adaptation of the healthy eating index to the food guide of the Brazilian population}

João Felipe MOTA²

Ana Elisa Madalena RINALDI²

Avany Fernandes PEREIRA ${ }^{3}$

Nailza MAESTÁ2

Marita Mecca SCARPIN²

Roberto Carlos BURINI ${ }^{2}$

\section{RE S U M O}

\section{Objetivo}

Adaptar o Índice de Alimentação Saudável norte-americano de acordo com as recomendações do Guia Alimentar da População Brasileira e da Pirâmide Alimentar Adaptada.

\section{Métodos}

Foi realizada avaliação do consumo alimentar de 502 indivíduos, de ambos os sexos (54, desvio-padrão=10 anos), na cidade de Botucatu (SP) ano de 2006. O método utilizado para estimar a ingestão foi o questionário recordatório de 24 horas e os alimentos relatados foram convertidos em porções, pelo valor energético, de acordo com os grupos alimentares da Pirâmide Alimentar Adaptada. Além disso, foram avaliados os nutrientes (colesterol, gordura total e saturada) e variedade da dieta. Foi realizada análise estatística descritiva e os valores intermediários da pontuação dos componentes foram calculados por razão e proporção.

\section{Resultados}

Foram alterados os números das porções alimentares conforme as estabelecidas na Pirâmide Alimentar Adaptada, além da inclusão dos grupos das leguminosas, doces e açúcares, óleos e gorduras na pontuação do Índice de Alimentação Saudável adaptado e exclusão do item sódio. Para os nutrientes a variedade da dieta foi

\footnotetext{
1 Artigo elaborado a partir da dissertação de J.F. MOTA, intitulada "Determinantes antropométricos, dietéticos, bioquímicos e físicos (aptidões aeróbia e muscular) da resistência insulínica". Programa de Pós-Graduação em Patologia, Faculdade de Medicina de Botucatu, Universidade Estadual Paulista; 2007

2 Universidade Estadual Paulista Júlio de Mesquita Filho, Faculdade de Medicina de Botucatu, Departamento de Saúde Pública, Centro de Metabolismo em Exercício e Nutrição. Distrito de Rubião Júnior, s/n., 18618-970, Botucatu, SP, Brasil. Correspondência para/Correspondence to: J.F. Mota. E-mail: <jfemota@yahoo.com.br>.

3 Universidade Federal do Rio de Janeiro, Instituto de Nutrição. Rio de Janeiro, RJ, Brasil.
} 
546 | J.F. MOTA et al.

estabelecida pontuação intermediária. O Índice de Alimentação Saudável adaptado pontuou 12 componentes no total, classificando as dietas em boa qualidade (boa qualidade: superior a 100 pontos), precisando melhorar (precisando melhorar: 71-100 pontos) e má qualidade (má qualidade: inferior a 71 pontos). A proporção de indivíduos classificados com dieta de boa qualidade 15\%, precisando melhorar $71 \%$ e de má qualidade $14 \%$.

\section{Conclusão}

O Índice de Alimentação Saudável adaptado é um instrumento que pode ser utilizado para avaliar os hábitos alimentares populacionais, porém específico para a população brasileira adulta. Contudo, se faz necessário que estes índices sejam constantemente revisados e adequados às novas recomendações nutricionais.

Termos de indexação: Avaliação nutricional. Hábitos alimentares. Nutrição. Registros de dieta.

\section{A B S T R A C T}

\section{Objective}

The objective of this study was to adapt the North American Healthy Eating Index according to the Dietary Guide of the Brazilian Population recommendations and the Adapted Food Pyramid.

\section{Methods}

Food intake of 502 individuals of both genders (mean age of 54 years, standard deviation of 10 years) was assessed in the city of Botucatu (SP) in 2006. Food intake was determined by the 24-hour recall and the reported foods were converted into portions according to the energy content and food groups of the Adapted Food Pyramid. Moreover, nutrients (cholesterol, total and saturated fat) and diet variety were also assessed. Descriptive statistical analysis was done and the intermediate values of the component scores were calculated by ratio and proportion.

\section{Results}

The number of food portions was modified according to the Adapted Food Pyramid, besides including the groups of legumes, sweets and sugars, oils and fats in the Adapted Healthy Eating Index score and excluding sodium. An intermediate score was established for nutrients and diet variety. The Adapted Healthy Eating Index scored a total of 12 components, classifying the diets as good quality (more than 100 points), needs improvement (from 71 to 100 points) and poor quality (under 71 points). The diet of 15\% of the individuals was classified as good, $71 \%$ needed improvement and $14 \%$ was poor.

\section{Conclusion}

The Adapted Healthy Eating Index is an instrument that can be used to assess population eating habits but it is specific for the adult Brazilian population. However, these indices need constant reviews and adaptations to new dietary recommendations.

Indexing terms: Nutritional assessment. Food habits. Nutrition. Diet records.

\section{N T R O D U ÇÃ O}

A partir da década de 80, a preocupação até então voltada à desnutrição associou-se a outro problema nutricional em expansão, a obesidade e suas conseqüentes comorbidades ${ }^{1}$. As ações de saúde pública voltadas principalmente para suprir o déficit nutricional, também se voltaram à elaboração de diretrizes alimentares para prevenção das doenças crônicas não-transmissíveis (DCNT). Diversos estudos mostram que melhorar a alimentação e, conseqüentemente, o estado nutricional é a chave para proporcionar benefícios à saúde da população e reduzir gastos públicos².
Pesquisas com características epidemiológicas buscam desenvolver métodos seguros e práticos que avaliem a qualidade da alimentação da população, bem como sua relação com as $\mathrm{DCNT}^{3}$. Contudo, os métodos disponíveis apresentam limitações significativas, pois analisam somente nutrientes isolados ou alguns grupos alimentares, fatos que inviabilizam considerar a complexidade do hábito alimentar de indivíduos e/ou populações ${ }^{3}$.

Segundo Hann et al. ${ }^{4}$, a avaliação isolada de nutrientes não traduz a natureza multifatorial da dieta humana. Além disso, a abordagem 
científica ao avaliar a quantidade de nutrientes e seus efeitos na saúde dos indivíduos, geralmente, apresenta a desvantagem de analisar a dieta separadamente do contexto sociocultural ${ }^{5}$.

Dessa forma, pode-se perceber a necessidade de elaborar instrumentos que avaliem o padrão alimentar de modo global, considerando tanto o consumo de nutrientes como de alimentos, refletindo em uma única variável a situação de diversos componentes da dieta ${ }^{6,7}$.

Com o intuito de conhecer os hábitos alimentares americanos e a adequação dos mesmos às diretrizes dietéticas estabelecidas no US Dietary Guidelines for Americans ${ }^{8}$, o Departamento de Agricultura dos Estados Unidos (USDA, 1989-1990) elaborou um índice para avaliar a qualidade global da dieta americana. Tal instrumento foi denominado Índice de Alimentação Saudável (Healthy Eating Index) e objetiva estimar a qualidade da dieta e também se propõe a avaliar o impacto de ações para intervenção nutricional ${ }^{2}$.

O índice de alimentação saudável avalia 10 componentes, sendo que 5 referem-se aos grupos alimentares da Pirâmide Alimentar americana ${ }^{9}, 4$ aos nutrientes (gordura total, saturada, colesterol, sódio) e 1 à medida da variedade da dieta. Cada componente apresenta um escore que varia de zero a 10, totalizando pontuação máxima 100 que se refere a uma dieta de boa qualidade $^{10}$.

Hann et al. ${ }^{4}$ mostraram que pontuações mais altas do índice de alimentação saudável associam-se de forma significativa à variedade da dieta, ao consumo aumentado de frutas, à adequação na ingestão de fibra dietética, ao baixo consumo de gordura total e saturada e às concentrações elevadas de alguns carotenóides e vitamina C. Além disso, foi encontrada influência positiva da idade, do nível educacional e da renda na pontuação mais alta do índice de alimentação saudável. Weinstein et al. ${ }^{3}$ observaram correlação positiva entre o índice de alimentação saudável e biomarcadores do consumo de frutas e hortaliças (vitamina $\mathrm{C}$, carotenóides, vitamina $\mathrm{E}$ e folato séricos).
Como os hábitos alimentares da população brasileira diferem em alguns aspectos dos americanos, o objetivo deste estudo foi adaptar o Índice de Alimentação Saudável norte-americano ao Guia Alimentar para a População Brasileira e à Pirâmide Alimentar Adaptada.

\section{MÉ TOD OS}

Após levantamento bibliográfico e estudos comparativos ${ }^{3,6,7,11}$ sobre índices dietéticos, optouse pela utilização do índice de alimentação saudável como base para adaptação ao Guia Alimentar para a População Brasileira. O índice de alimentação saudável apresenta como vantagens a avaliação do consumo de grupos alimentares ao invés de nutrientes, fornecendo resultados mais facilmente aplicáveis à avaliação dietética e pode ser utilizado para indivíduos a partir de dois anos de idade. Além disso, o índice de alimentação saudável é o método que melhor se correlaciona a variáveis representativas de uma dieta adequada ${ }^{4}$.

Em deferência às orientações alimentares, representadas pela Pirâmide Alimentar Adaptada brasileira'12 e o Guia Alimentar para a População Brasileira $^{13}$, foi realizada a adaptação do índice de alimentação saudável americano (IASad) ${ }^{14,15}$.

Para o cálculo do IASad foi realizada avaliação do consumo alimentar, no ano de 2006, de 502 indivíduos, de ambos os sexos (142 homens; 364 mulheres), com média (M) de idade de 54 (desvio-padrão - DP=10) anos, participantes do Projeto de Extensão universitária "Mexa-se Pró-Saúde" oferecido pelo Centro de Metabolismo em Exercício de Nutrição (CeMENutri), da Faculdade de Medicina de Botucatu (FMB/Unesp). O método utilizado para estimar a ingestão foi o método recordatório de 24 horas, totalizando quatro questionários por indivíduo ${ }^{16}$. Com o intuito de melhorar a estimativa da quantidade consumida por cada indivíduo, utilizou-se o registro fotográfico ${ }^{17}$.

Assim sendo, os dados dietéticos obtidos em medidas caseiras foram convertidos para 
grama e mililitro, a fim de possibilitar a análise química do consumo alimentar, e as informações foram processadas por meio do programa de análise nutricional NutWin, versão $1.5^{18}$. Os alimentos não disponíveis no programa foram acrescentados, posteriormente, seguindo-se a seqüência de tabelas para entrada de dados: Tabela Brasileira de Composição de Alimentos (TACO) ${ }^{19}$, Tabela de Composição de Alimentos ${ }^{20}$, Tabela de Composição de Alimentos: Suporte para Decisão Nutricional21, Tabela para Avaliação de Consumo Alimentar em Medidas Caseiras ${ }^{22}$. Como o número de alimentos aumenta rapidamente no mercado, algumas informações nutricionais e de peso dos alimentos foram obtidas a partir dos rótulos dos mesmos.

Também com base no recordatório de 24 horas, os alimentos relatados foram convertidos em porções pelo valor energético, de acordo com o grupo ao qual pertencem: cereais, frutas, hortaliças, leguminosas, produtos lácteos, carnes, doces e açúcares, óleos e gorduras (Quadro 1) ${ }^{13}$.

As preparações culinárias elaboradas com mais de um grupo alimentar foram desmembradas nos seus ingredientes e estes classificados nos respectivos grupos correspondentes. Este procedimento segue as recomendações da Pirâmide Alimentar Adaptada ${ }^{12}$.

As adaptações realizadas foram alterações do número das porções alimentares conforme as estabelecidas na Pirâmide Alimentar Adaptada ${ }^{12}$,

Quadro 1. Valor energético equivalente a uma porção dos grupos alimentares da Pirâmide Alimentar Brasileira adaptada.

\begin{tabular}{lc}
\hline Grupos alimentares da pirâmide adaptada & Valor energético (kcal) \\
\hline Cereais, pães, raízes e tubérculos & 150 \\
Hortaliças & 15 \\
Frutas & 70 \\
Leguminosas e oleaginosas & 55 \\
Carnes e ovos & 190 \\
Leite e produtos lácteos & 120 \\
Óleos e gorduras & 73 \\
Açúcares e doces & 110 \\
\hline
\end{tabular}

Adaptado de Philippi'2 incluindo os grupos das leguminosas, açúcares e gorduras na pontuação do IASad e excluindo sódio, devido à dificuldade na estimativa de consumo deste micronutriente e deficiência de dados disponíveis nas tabelas de composição de alimentos brasileiras.

Além disso, foram avaliados os nutrientes (gordura total, saturada e colesterol) conforme a metodologia do índice de alimentação saudável norte-americano. O último componente avaliado foi a variedade do consumo diário de alimentos. A partir do recordatório de 24 horas foram contabilizados todos os diferentes alimentos consumidos. Porém, o mesmo alimento preparado de diferentes formas (cozido, assado ou frito) foi computado apenas uma vez ${ }^{2}$.

O estudo foi conduzido dentro dos padrões exigidos pela Declaração de Helsinki (1975), modificada em 1983 e aprovado pela comissão de ética em pesquisa da Faculdade de Medicina de Botucatu, Brasil (OF. 170/2005 - CEP). Todos os indivíduos assinaram o termo de consentimento livre e esclarecido.

Para análise dos dados foi realizada estatística descritiva, utilizando o software Statistica for Windows (version 6.0, Statsoft, Tulsa, USA). $\mathrm{Na}$ adaptação do índice de alimentação saudável os valores intermediários da pontuação dos componentes foram calculados por razão e proporção.

RES ULTADOS

A pontuação dos componentes do índice de alimentação saudável adaptado variou de 0 a 10 (Tabela 1), sendo que os valores intermediários foram calculados proporcionalmente. Dessa forma, quando o consumo dos grupos alimentares fosse igual ou superior ao recomendado atribuiram-se 10 pontos e quando inferior os pontos foram calculados por razão e proporção.

Para os nutrientes (gordura total, saturada e colesterol) e a variedade, foi criada pontuação proporcional em relação ao que era consumido. 
Tabela 1. Componentes do Índice de Alimentação Saudável adaptado de Kennedy² e o respectivo critério de pontuação. Botucatu, (SP), 2006.

\begin{tabular}{lccc}
\hline & Pontuação* & Pontuação máxima de 10 & Pontuação mínima de 0 \\
\hline Grupo dos cereais, pães, tubérculos e raízes & 0 a 10 & 5 - 9 porções & 0 porções \\
Grupo dos vegetais & 0 a 10 & 4 - 5 porções & 0 porções \\
Grupo das frutas & 0 a 10 & 3 - 5 porções & 0 porções \\
Grupo das leguminosas & 0 a 10 & 1 porção & 0 porções \\
Grupo das carnes & 0 a 10 & $1-2$ porções & 0 porções \\
Grupo dos laticínios & 0 a 10 & 3 porções & 0 porções \\
Grupo dos óleos e gorduras & 0 a 10 & $1-2$ porções & 0 porções \\
Grupo dos açúcares e doces & 0 a 10 & $1-2$ porções & 0 porções \\
Gordura total (\%) & 0 a 10 & $\leq 30$ & $\geq 45$ \\
Gordura saturada (\%) & 0 a 10 & $<10$ & $\geq 15$ \\
Colesterol alimentar (mg) & 0 a 10 & $\leq 300$ & $\geq 450$ \\
Variedade & 0 a 10 & $\geq 8$ diferentes itens/dia & $\leq 3$ diferentes itens/dia \\
\hline
\end{tabular}

*Pessoas com o consumo ou a ingestão entre o limite máximo e o mínimo receberam pontuações proporcionais (regra de três).

Dessa forma, para o consumo de gordura total foi estabelecido o valor entre $31,0 \%-44,9 \%$, saturada entre 10,0\%-14,0\%, colesterol entre 300-449mg, e para a variedade entre 4-7 itens diferentes/dia foram atribuídos cinco pontos.

Assim, de acordo com o IASad foram pontuados 12 componentes no total, sendo 8 referentes aos grupos alimentares da Pirâmide Alimentar Adaptada brasileira; 3 nutrientes (gordura total, saturada e colesterol) e variedade de alimentos conforme o proposto pelo índice de alimentação saudável americano (Tabela 1).

Logo, a partir da pontuação obtida das variáveis avaliadas no IASad, recomenda-se que as dietas sejam classificadas em boa qualidade (superior a 100 pontos), precisando de melhorias (71-100 pontos) e má qualidade (inferior a 71 pontos).

Dos 502 indivíduos avaliados, 15\% $(n=76)$ apresentaram dieta de boa qualidade, $71 \%$ $(n=356)$ precisando de melhorias e $14 \%(n=70)$ dieta de má qualidade. As médias da pontuação do IASad podem ser observadas na Tabela 2.

\section{I S CUSS Ã O}

A adaptação do índice mostrou-se necessária pelo fato de o Guia Alimentar para População Brasileira diferir significativamente do
Tabela 2. Média (M) e desvio-padrão (DP) da pontuação do Índice de Alimentação Saudável adaptado de Kennedy². Botucatu (SP), 2006.

\begin{tabular}{lccc}
\hline \multirow{2}{*}{ Dietas } & $\mathrm{n}$ & \multicolumn{3}{c}{ Pontuação do IASad } \\
\cline { 3 - 4 } & 502 & 86,7 & 14,1 \\
\hline Geral & 70 & 62,2 & 6,4 \\
Dieta de má qualidade & 356 & 87,2 & 7,8 \\
Dieta precisando de melhorias & 76 & 106,8 & 4,7 \\
Dieta de boa qualidade & 76
\end{tabular}

*IASad: índice de alimentação saudável adaptado.

americano. Como o Índice de Alimentação Saudável inclui em sua pontuação as porções alimentares estabelecidas no US Dietary Guidelines for Americans $^{8}$ e na Pirâmide Alimentar americana ${ }^{9}$, optou-se por utilizar as recomendações das porções estabelecidas no Guia Alimentar para a População Brasileira ${ }^{13}$ e na Pirâmide Alimentar Adaptada².

Outro fator importante é que apesar de a nova Pirâmide Alimentar americana estabelecer as recomendações para os grupos de óleos e gorduras e açúcares e doces, esta não estabelece a quantidade de energia equivalente a uma porção, como referencia a Pirâmide Alimentar Adaptada. Além disso, na Pirâmide Alimentar americana as leguminosas pertencem ao grupo das carnes e ovos. Por fazerem parte do hábito alimentar brasileiro, apresentarem porções 
específicas recomendadas e contribuírem para o consumo de proteínas, as leguminosas foram pontuadas à parte.

Em 2004, Fisberg et al. ${ }^{11}$ fizeram uma adaptação do Índice de Qualidade da Dieta (IQD), utilizando as porções recomendadas pela Pirâmide Alimentar Adaptada, porém sem pontuar os grupos alimentares leguminosas, açúcares e doces, óleos e gorduras.

Neste estudo, além de contabilizados os grupos alimentares dos cereais, hortaliças, frutas e laticínios, foram acrescentados os grupos das leguminosas, açúcares e doces, óleos e gorduras, de acordo com as recomendações do Guia Alimentar para a População Brasileira ${ }^{13}$. As recomendações para o consumo de gorduras totais, saturadas e colesterol não foram alteradas, pois seguem as mesmas recomendações propostas pelo US Dietary Guidelines for Americans ${ }^{8}$.

A principal limitação do estudo foi a necessidade de excluir o sódio dietético da pontuação do IASad, uma vez que a estimativa de consumo de sódio utilizado no preparo dos alimentos é extremamente difícil e pouco precisa, além de haver deficiência de dados disponíveis do teor deste mineral nas tabelas de composição de alimentos brasileiras ${ }^{23-25}$. Para atribuição de pontos à variedade dos alimentos consumidos no período de um dia, foram utilizadas as recomendações propostas pelo índice de alimentação saudável².

A pontuação do IASad se diferenciou daquela do índice de alimentação saudável, que classificava o consumo alimentar dos sujeitos analisados como dieta de má qualidade (inferior a 51 pontos), precisando de melhorias (51-80 pontos) e boa qualidade (superior a 80 pontos)². Tal alteração deve-se ao acréscimo de três novos componentes (grupos das leguminosas, açúcares e gorduras) e à exclusão do componente sódio (Tabela 3).

De acordo com a pontuação obtida, observou-se que a maioria dos indivíduos teve sua alimentação classificada como dieta precisando de melhorias. Recentemente, observou-se que a
Tabela 3. Comparação entre a pontuação final do Índice de Alimentação Saudável americano e o Índice de Alimentação Saudável adaptado de Kennedy². Botucatu (SP), 2006.

\begin{tabular}{lcc}
\hline \multirow{2}{*}{ Classificação da dieta } & \multicolumn{2}{c}{ Pontuação } \\
\cline { 2 - 3 } & IAS $^{*}$ & IASad $^{* *}$ \\
\hline Má qualidade & $<51$ & $<71$ \\
Precisando de melhorias & $51-80$ & $71-100$ \\
Boa qualidade & $>80$ & $>100$ \\
\hline
\end{tabular}

IAS: índice de alimentação saudável americano; IASad: índice de alimentação saudável adaptado.

*Total de componentes avaliados: 10; **Total de componentes avaliados: 12, em decorrência do acréscimo dos grupos das leguminosas, açúcares e doces, óleos e gorduras; e exclusão do componente sódio.

população do Estado de São Paulo apresenta inadequação no seu consumo alimentar ${ }^{26}$. Além disso, Bermudez \& Tucker $^{27}$ verificaram que a alimentação da população da América Latina é inadequada e está associada ao desenvolvimento das doenças crônicas não transmissíveis.

A criação de um índice com base em propostas específicas para a população brasileira, e que avalie de forma qualitativa e quantitativa a dieta habitual, faz-se necessária para propor intervenções dietéticas mais eficazes. O IASad é um instrumento que pode ser utilizado para avaliar os hábitos alimentares populacionais, porém é específico para a população brasileira. Contudo, é necessário que estes índices sejam constantemente revisados e adequados às novas recomendações nutricionais.

\section{A GRADECIMENTOS}

Aos alunos de iniciação científica e aprimoramento: Olívia Lucena de Medeiros; Fabio Fabian Buscariollo; Letícia Teixeira Rocio; Fabiana Denipote; Fabiana Marzolla; Viviane Sakezenian; Carolina Sartori; Juliana Machado Bastos; Helena Siqueira Vassimon; Cristina Pichinin Grespan (in memorian). Ao Conselho Nacional de Desenvolvimento Científico e Tecnológico (CNPq), pela bolsa concedida.

\section{COLABORADORES}

J.F. MOTA responsável pela condução do projeto de pesquisa, pela avaliação nutricional dos 
indivíduos participantes, pela análise dos dados e pela redação do manuscrito. A.E.M. RINALDI colaborou na avaliação nutricional dos indivíduos participantes, participou da análise dos dados e da redação do manuscrito. A.F. PEREIRA responsável pela condução do projeto de pesquisa, colaborou na avaliação nutricional dos indivíduos participantes, participou da análise dos dados e da redação do manuscrito. N. MAESTÁ responsável pela condução do projeto de pesquisa, colaborou na avaliação nutricional dos indivíduos participantes, participou da análise dos dados e da redação do manuscrito. M.M. SCARPIN colaborou da avaliação nutricional dos indivíduos participantes, participou na análise dos dados e da redação do manuscrito. R.C. BURINI orientador do projeto de pesquisa, responsável pela revisão da análise dos dados, colaborou na redação final do trabalho.

\section{REFER Ê N CIAS}

1. Zeferino $A M B$, Filho $A A B$, Bettiol $H$, Barbieri MA. Acompanhamento do crescimento. J Pediatr. 2003, 79(1):S23-S32.

2. Kennedy ET, Ohls J, Carlson S, Fleming K. The Healthy Eating Index: design and applications. J Am Diet Assoc. 1995; 95(10):1103-9.

3. Weinstein SJ, Vogt TM, Gerrior SA. Healthy Eating Index scores are associated with blood nutrient concentrations in the Third National Health and Nutrition Examination Survey. J Diet Assoc. 2004; 104(4):576-84.

4. Hann CS, Rock CL, King I, Drewnowski A. Validation of the Healthy Eating Index with use of plasma biomarkers in a clinical sample of women. Am J Clin Nutr. 2001; 74(4):479-86.

5. Mertz W. Foods and nutrients. J Am Diet Assoc. 1984; 84(1):769-70.

6. Vieira VL, Gomes ALC, Araújo EA, Cervato AM. Qualidade da dieta: avaliação por meio de dois instrumentos de medida. Rev Bras Nutr Clin. 2005; 20(1):9-15

7. Cervato AM, Vieira VL. Índices dietéticos na avaliação da qualidade global da dieta. Rev Nutr. 2003; 16(3):347-55.

8. Nutrition and Your Healthy. Dietary guidelines for americans. 3th ed. Washington (DC): US Department of Agriculture; 1999. Home and Garden Bulletin, nº 232.

9. U.S. Department of Agriculture. The food guide pyramid: a guide to daily food choices. Washington (DC): Center for Nutrition Policy and Promotion; 1992. Home and Garden Bulletin, $n^{\circ} 252$.
10. Bowman AS, Lino M, Gerrior AS, Basiotis PP. The healthy eating index: 1994-96. Washington (DC): US Department of Agriculture, 1998. Publication $\mathrm{n}^{\circ}$ CNPP-5.

11. Fisberg RM, Slater $B$, Barros RR, Lima FD, Cesar $C L G$, Carandina L, et al. Índice de qualidade da dieta: avaliação da adaptação e aplicabilidade. Rev Nutr. 2004; 17(3):301-8.

12. Philippi ST, Latterza AR, Cruz ATR, Ribeiro LC. Pirâmide alimentar adaptada: guia para escolha dos alimentos. Rev Nutr. 1999; 12(1):65-80.

13. Brasil. Ministério da Saúde. Secretaria de Atenção à Saúde. Coordenação-Geral da Política de Alimentação e Nutrição. Guia alimentar para a população brasileira: promovendo a alimentação saudável. Brasília: Ministério da Saúde; 2005.

14. Pereira AF, Morelli MYG, Mota JF, Sakzenian VM, Bastos JM, Vassimon HS, et al. Avaliação da qualidade da dieta de amostra populacional adulta de Botucatu segundo o índice de alimentação saudável (IAS) segundo as pirâmides alimentares brasileira e americana [abstract]. Rev Bras Nutr Clin. 2003; 18(1):S541.

15. Mota JF, Medeiros OL, Denipote FG, Buscariolo FF. Prevalência do consumo de frutas e hortaliças, associada à ingestão excessiva de gorduras em adultos de acordo com a faixa etária [abstract]. Gerontologia. 2004; 12(1):S56.

16. Crispim SP, Franceschini SCC, Priore SE, Fisberg RM. Validação de inquéritos dietéticos: uma revisão. Nutrire: Rev Soc Bras Alim Nutr. 2003; 26(12): 127-41.

17. Zabotto CB, Vianna RPT, Gil MF. Registro fotográfico para inquéritos dietéticos: utensílios e porções. Instituto Nacional de Alimentação e Nutrição (INAN). Goiânia: Núcleo de Estudos e Pesquisas em Alimentação, Universidade Estadual de Campinas; 1996.

18. Programa de Apoio à Nutrição. Versão 1.5. São Paulo: Departamento de Informática em Saúde, Universidade Federal de São Paulo; 2002.

19. Núcleo de Estudos e Pesquisas em Alimentação. Universidade Estadual de Campinas. Tabela brasileira de composição de alimentos. Versão II. 2a. ed. Campinas: Unicamp; 2006.

20. Instituto Brasileiro de Geografia e Estatística. Tabela de composição de alimentos. 4a. ed. Rio de Janeiro: IBGE; 1996.

21. Philippi ST. Tabela de composição de alimentos: suporte para decisão nutricional. 2a. ed. São Paulo: Coronário; 2002.

22. Pinheiro ABV, Lacerda EMA, Benzecry EH, Gomes MCS, Costa VM. Tabela para avaliação de consumo 
alimentar e medidas caseiras. São Paulo: Atheneu; 2002.

23. Alaimo K, McDowell MA, Briefel RR. Dietary intake of vitamins, minerals, and fiber of persons ages 23 months and over in the United States: third national health and nutrition examination survey, phase 1, 1988-91. Adv Data Vital and Health Statistics. 1994; 258:1-26.

24. Pennington JA. Bowes and Church's food values of portions commonly used. 16th ed. Philadelphia (PA): JB Lippincott Company; 1994.

25. Espeland MA, Kumanyika S, Wilson AC, Reboussin DM, Easter L, Self $M$, et al. Statistical issues in analyzing 24-Hour dietary recall and 24-hour urine collection data for sodium and potassium intakes. Am J Epidemiol. 2001; 153(10):996-1006.

26. Fisberg RM, Morimoto JM, Slater B, Barros MBA, Carandina L, Goldbaum MJ, et al. Dietary quality and associated factors among adults living in the State of São Paulo, Brazil. J Am Diet Assoc. 2006; 106(12):2067-72.

27. Bermudez OI, Tucker KL. Trends in dietary patterns of Latin American populations. Cad Saúde Pública. 2003; 19(Suppl):S87-99.

Recebido em: 13/4/2007

Versão final reapresentada em: 23/4/2008 Aprovado em: 28/5/2008 